Eliana Goldfarb Cyrino 1

Maria Lúcia Toralles Pereira 2

\title{
Reflexões sobre uma proposta de integração saúde-escola: o projeto saúde e educação de Botucatu, São Paulo
}

\author{
Integration of health into the school curriculum \\ in Botucatu, São Paulo
}

\footnotetext{
1 Departamento de Saúde Pública, Faculdade de Medicina de Botucatu, Universidade Estadual de São Paulo. Distrito de Rubião J r. s/no, C. P. 549, Botucatu, SP 18618-970, Brasil. dspaula@fmb.unesp.br 2 Departamento de Educação, Instituto de Biociências de Botucatu, Universidade Estadual de São Paulo. Distri to de Rubião Jr. s/no, C. P. 549 , Botucatu, SP 18618-970, Brasil. toralles@ibb.unesp.br
}

Abstract This study focuses on the drafting and development of a public heal th program aimed at strategi es to integrate heal th activities and the school curriculum. The program is based on three main lines of activity: full health care for schoolchildren, with special emphasis on the public school system; training of personnel in the fields of health and education by means of internships all owing participants to experience, work, and refl ect critically on the acti vities with an interdisciplinary team; and work wi th teachers from the public elementary school system to implement and devel op innovative measures in the fields of health and education to respond to the demand by schools and the community.

Key words Health Education; School Health; Health Care; Students

Resumo Tendo como objeto de estudo a criança em idadeescolar, discute-se, no presente artigo, o processo de elaboração e desenvolvi mento de uma programação em saúde pública a fim de construir uma estratégi a de ações que integrem os campos da saúde e da edu cação. Tal programação apresenta-se apoiada em três ei xos de atuação: atenção integral à saú de da criança em idade escolar, priorizando a cri ança que está ingressan do no si stema públi co escolar; formação de recursos humanos nas áreas saúde e educação, por meio de estági os que possi bilitem vivenciar, trabal har e refletir criticamente a ação programática junto a uma equipe interdisciplinar; trabal ho com professores da rede de ensino público fundamental para a implantação e desenvolvi mento de ações in ovadoras nos campos da saú de e da educação que respondam às demandas da escola, da comunidade e de uma atuação crítica em face dos probl emas enfrentados pelas crianças em processo de escolarização.

Palavras-chave Educação em Saúde; Saúde Escolar; Cuidados de Saúde; Estudantes 
A saúde da criança em face das políticas de saúde e educação

A preocupação do Brasil com a proteção à infância e à maternidade em termos formais data de 1921, quando, por ocasião da reorganização dos serviços de saúde, conhecida como Reforma Carlos Chagas, o governo assumiu a responsabilidade de atuar na área da higiene infantil. Somente em 1975, porém, éque se introduzem medidas concretas para articular o atendimento à saúde da mulher e da criança entre as três esferas do poder público - federal, estadual e municipal - e o setor privado, mediante a Lei n. 6.229, que dispõe sobre a organização do sistema nacional de saúde e a reorganização da assistência à saúde no âmbito da Previdência Social (Yunes et al., 1987).

Após a Constituição Federal de 1988, o grande desafio para as administrações municipais, na área da saúde, tem sido a reorganização dos serviços, tendo em vista a construção do Sistema Ú nico de Saúde (SUS).

Contemplar os princípios do SUS de regionalização, descentralização com direção única em cada esfera de governo, acesso universal, atendimento integral com prioridade para as ações preventivas e participação da comunidade, implica, também, rever a questão da atenção à saúde da criança. Para al gumas parcelas da população infantil, como a criança em idade escolar ou o adolescente, chama-se a atenção para a marginalização ao atendimento realizado pelo setor saúde, a necessi dade de aumentar a cobertura e melhorar a qualidade dos serviços oferecidos a essa população.

Pode-se dizer que os anos 80 significaram para as administrações municipais no Brasil um momento de reordenação, de maior possibilidade de desenvolvimento de propostas e de ampliação dos espaços democráticos de participação. Assim, em Botucatu, cidade do interior de São Paulo, inicia-se, a partir de 1983, a organização e implantação de um serviço municipal de saúde, tendo como meta de seu plano de ação a implantação de uma rede básica de serviços de atenção primária com integração aos diversos níveis de complexidade.

$\mathrm{Na}$ organização do trabalho, a preocupação de realizar estudos que buscassem uma análise da realidade da saú de da população marcou o momento de implantação dos serviços. Tornouse necessário rever modelos e trabalhar com problemas de saúde estabelecidos pelas condições de vida de grupos populacionais específicos e por processos mais abrangentes da sociedade.

Dentro desse movimento, inicia-se, a partir de 1985, uma programação em saúde voltada à criança em idade escolar no município de Botucatu.

Naquele momento, constatava-se que, no caso da criança em idade escolar, que freqüentava ou não a escola pública de ensino fundamental (antigo primeiro grau), havia uma grande ausência de serviços de saúde voltados às necessidades desse grupo populacional. Talvez, excetuando-se al gumas condições específicas, tal grupo etário, que apresenta um baixo grau de objetivação do ponto de vista do instrumento clássico da Medicina, pouco utilizasse os serviços de saúde, porque eram poucas as necessidades interpretadas pelos serviços de saúde (Ayres, 1990).

Ressalta-se, assim, a presença de um quadro de morbidade não valorizado nas estatísticas de saúde, como os problemas psicossociais, gravidez na adolescência, queixas psicossomáticas, doenças sexual mente transmissíveis, cáries, distúrbios de visão, o problema do uso de drogas, entre outros, como uma demanda freqüente de problemáticas da criança aos serviços de saúde e para os quais as Unidades de Saúde não estavam organizadas para dar respostas (SMSSP, 1990).

A necessidade de buscar uma visão integradora do processo de crescimento e desenvolvimento de nossa infância e adolescência vem sendo apontada, incluindo aí a leitura sociológica, antropológica e política que justifica seus comportamentos, sua expressão no corpo biológico e seus planos de metas que organizam um modo de vida saudável ou de risco. As ações básicas de saúde, consagradas pela Organização Mundial da Saúde, foram capazes de reduzir a mortalidade de jovens e crianças e têm sido capazes de conter até $60 \%$ das mortes no primeiro ano de vida, mas ressalta-se que a criança, vista na sua integralidade, ainda está fora do sistema de saúde. Os maiores de cinco anos estão fora das prioridades em saúde, pelo menos em suas prioridades específicas (Ceccin et al., 1992; Lima, s/d).

Por outro lado, na década de 80 , houve um crescimento expressivo da taxa de escol arização para a população de sete a 14 anos em todo o país. Apesar desse crescimento, a década de 90 começa com aproximadamente quatro milhões de crianças em idade escolar que não freqüentam a escola. Dado que uma parcela significativa destas crianças já freqüentara, em algum período de suas vidas, a escola, mas foram posteriormente excluídas dela, poder-seia dizer que a ampliação de vagas das escolas públicas no Brasil ainda ap resenta problemas que precisam ser melhor esclarecidos.

Assim, se a década de 80 significou ao Estado brasileiro um momento de ressurgimento 
de movimentos pela democratização da sociedade, com profundos reflexos nos caminhos dos setores saú de e educação, há de selevar em conta o expressivo aumento de demanda em ambas as áreas, ou seja, nos dois referidos setores foi possível observar grande extensão da cobertura, com conseqüências que precisam ser avaliadas e estudadas.

\section{Saúde escolar: crítica ao recorte}

A análise dos programas de saúde escolar desenvolvidos no Brasil, ainda hoje, permitiu observar que, embora preocupados com uma ação ampla, de modo geral, eles têm, na prática, uma linha assistencialista com predomínio de subprogramas isolados, como a assistência odontológica, oftalmológica e psicológica. Ou seja, a saúde escolar reproduz o paradigma de caráter assistencialista da atenção em saúde em geral, que prioriza o indivíduo e partes dele, em detrimento da coletividade e do todo (Sucupira et al., 1989).

Atualmente, existem grupos que trabalham com a saúde escolar, ligando-a a questões mais específicas do comportamento e das síndromes e disfunções cerebrais, assim como das carências nutricionais e da pobreza. A possibilidade de outros recortes que se aproximem mais do eixo das principais necessidades da criança nessa faixa etária justifica a necessidade de maior participação nesse campo do conhecimento, com a criação de trabal hos que se contraponham, no plano técnico-científico, ético e político, à visão defendida pela porção, ainda hegemônica nessa área, de que a miséria da população é a causa de seus males e fracassos (Fonseca, 1988). A conseqüência dessa visão nos espaços escolares resulta, freqüentemente, num etnocentrismo das organizações educativas, que se preocupam, rapidamente, em identificar as inúmeras faltas nas crianças das camadas populares: falta de inteligência, falta de um ambiente familiar adequado, falta de conhecimentos anteriores, falta de maturidade para serem alfabetizadas, falta de condições nutricionais, falta de saúde etc. E assim, como seres de carência absoluta, o destino dessas crianças já está traçado pela profecia auto-realizável do professor, dos especialistas e da sociedade que antecipam, an unciam e acabam contribuindo para a produção do fracasso dessa população (Patto, 1990).

Com movimentos ambíguos e contraditórios, essa profecia ora volta-se para uma prática assistencialista, ora para uma prática de descompromisso e injustiça social em face dos di- reitos dessa criança, contribuindo para que, muito rapidamente, ela se perceba culpada de seu próprio fracasso na escola e na sociedade.

O projeto de integração saúde

e educação em Botucatu

Nesse contexto de discussão e preocupações, e com base numa visão ampliada de saúde, teve início, no final de 1985, o Programa de Saúde Escolar (PSE) de Botucatu, a partir da reivindicação de uma escola estadual da periferia da cidade.

A proposta de ação inicial objetivava, basicamente, realizar um diagnóstico da situação de saúde e do processo educacional dos escolares, identificar as necessidades do corpo docente da referida escola e delinear uma proposta de ação com a participação de todos os envolvidos (professores, alunos, familiares).

Para o desenvolvimento do projeto inicial, gradativamente formou-se uma equipe de profissionais das áreas saúde/ educação (profissionais do Serviço Municipal de Saúde, do Departamento de Saúde Pública e Departamento de Neurologia e Psiquiatria da Faculdade de Medicina de Botucatu - FMB - e do Centro de Saúde Escola), com a preocupação de compreender os problemas que envolvem a saúde da criança ingressante no sistema escolar e de construir propostas capazes de responder a esses problemas.

Priorizando a necessidade de afirmar um espaço de reflexão permanente e de construção de conhecimentos na área da saúde da criança em processo de escolarização, o trabaIho exigiu perceber o movimento entre ação e reflexão, o que trouxe necessi dade de mudanças de estratégias para o enfrentamento dos problemas emergentes. Esse processo foi também subsidiado pela análise crítica de programas de saúde escolar desenvolvidos anteriormente, seja no âmbito nacional, seja no Município de Botucatu.

Esse trabalho estruturou-se como uma programação em saúde pública, na qual os objetivos não se limitaram ao atendimento imediato dos problemas médicos do escolar nem se restringiram ao atendimento de demandas individuais e/ ou espontâneas, mas voltaram-se para assegurar o direito da criança de ben eficiar-se de ações organizadas no plano coletivo. Desta forma procurava-se possibilitar uma maior integração entre equipe de saúde, escola, criança e família, objetivando contribuir para assegurar o direito à saúde e à educação no processo de escolarização. 
Na avaliação do trabalho inicial, foi possível observar que a presença da equipe no ambiente escolar, trazendo uma leitura crítica dos programas de saúde escolar já desenvolvidose, ao mesmo tempo, acenando para esta com uma nova proposta, parece ter deflagrado uma série de conflitos e insatisfações, de ambas as partes, revelando, desde o início, que o trabalho conjunto não se desenvolveria num espaço de harmonia. As tensões e contradições precisariam ser aceitas para que a comunicação entre equipe, escola, crianças e familiares pudesse efetivar um processo de construção participativa, tendo como base os problemas identificados e priorizados por todos. Acostumados a uma presença episódica do setor saúde, reivindicando soluções para questões médicas essencialmente curativas ou mesmo não médicas, porém interpretadas pela ótica “medicalizadora", direção e professores depararam com uma equipe desejosa de repensar a saúde escolar pela integração saúde e educação, buscando novas propostas na detecção e solução dos problemas, que, muitas vezes, extrapolavam a ambos setores.

A constatação de que o setor saúde depara com limitações, tendo em vista a perspectiva medicalizadora, assim interpretada pela escola, dos problemas educacionais das crianças das camadas populares, dificultou a integração inicial entre equipe de saú de e professores. Isso pôde ser observado, sobretudo, no momento em que se levantou a questão da não-correspondência direta entre as dificuldades do processo de aprendizagem e causas médicas ou condições gerais de vida de alguns escolares. $\mathrm{Na}$ medida em que a equipe explicitava suas idéias, discutindo que, apesar da pobreza, das condições extremamente adversas no ambiente familiar, tanto a educação, como a saúde, tinham um papel a desempenhar para o desenvolvimento das potencialidades das crianças consolidando a auto-estima de cada criança e, ao mesmo tempo, criando novos desafios para ampliar o seu conhecimento inicial -, observavam-se obstáculos que dificultavam o avanço da proposta de um trabalho conjunto de construção e reflexão de conhecimentos nessa área.

Outras estratégias de atuação foram sendo construídas e o PSE buscou, também, uma nova forma de inserção dentro das idéias gerais de reformulação do setor saúde, procurando trabalhar a atenção à saúde da criança em idade escolar, num padrão de assistência cujo enfoque maior voltou-se para a compreensão, orientação e ação ampla sobre o processo saúde-doença e seus determinantes. Procurou-se desenvolver ações baseadas na análise das ne- cessidades detectadas na escola, propondo-se um modelo de atendimento que, de certa maneira, entrava em conflito com a solicitação desta, mas que respondia a algumas de suas demandas. A preocupação nesse momento era trabalhar na dialética continuidade-ruptura, consolidando ações desenvolvidas no interior da escola, criando, no entanto, sempre novos desafios por meio da atuação da equipe com grupos de crianças.

Nessa etapa do trabalho, chamou a atenção o fato de a escola ter encaminhado à consulta médica preferencialmente escolares vistos como fracassados e crianças com problemas de pele, ou seja, nesse grupo, dez crianças (35\%) com queixa de repetência ou mal rendimento escolar foram encaminhadas, por serem consideradas pela escola com maior prioridade para atendimento médico. Quanto às demais queixas, referiam-se principalmente a problemas de pele, visão, comportamento, dores, problemas, talvez mais relacionados à aparência dos escolares (Cyrino, 1994:84-85).

Procurando discutir a deman da da escola, a equipe do PSE buscava novos espaços para a construção de conhecimentos que revertessem em mudanças de atitude e mudanças metodológicas na atuação prática com crianças escolares. Nesse momento, a necessidade de uma concepção interdisciplinar para enfrentar a complexidade dos problemas concretos exigiu um trabal ho coletivo de reflexão e estudo organizado valendo-se de problemas comunse integradores, por meio de leitura e análise da prática e de discussão de textos (tais como Sucupira et al.,1986; Uribe-Rivera, 1989; Valla, 1989; Gonçalves et al., 1990; Schraiber, 1990, 1992; Castellanos, 1991, no campo da saúde, e Patto, 1990; Ferreiro, 1986; Vygotsky, 1987; Freire \& Faundes, 1985, no campo da educação). Textos que proporcionavam a problematização de conhecimentos necessários à equipe para fundamentar uma prática consciente e crítica em relação aos pressupostos políticos, éticos e epistemológi cos da atuação pedagógica nos campos da saúde e da educação.

A composição da equipe, com profissionais vindos de diferentes áreas - saúde mental, educação, saú de pública - facilitou a construção desse trabalho coletivo voltado para diferentes práticas que pretendiam renovar-se pela incorporação de uma visão mais ampla dos problemas que envolvem as crianças das camadas populares em seu processo de escolarização.

Para a equipe do PSE, ficava evidente a necessidade de trabalhar com a escola numa proposta de parceria em que a segunda se abrisse para construir com a primeira novos desafios, 
num processo de participação ativa de todos, capaz de tornar conscientes os limites de cada um (equipe e escola), percebendo a própria transitoriedade desses limites, para mudar as relações com a população: escolares e comunidade. Para isso, era preciso que a escola fosse transformada em um espaço de comunicação em todas as direções, incentivando o diálogo e a leitura crítica da prática, resgatando o prazer de descobrir e conhecer num processo cooperativo e participativo, fortalecendo a autoconfiança nas próprias potencialidades e encorajando o desafio para ampliar o conhecimento e superar os próprios limites.

Do ponto de vista metodológico, esse foi o grande desafio da equipe, tendo em vista os conflitos dessa convivência comunicativa, revelados já nos primeiros encontros na escola, nas expectativas dos professores e direção em relação ao trabalho da equipe.

\section{Ampliando os espaços de atuação}

O compromisso da equipe com as idéias que estruturavam a proposta favoreceu a permanente busca de estratégi as para a continuidade da mesma. O PSE foi, gradativamente, ampliando-se e incorporando novas proposições, não sem conflitos e dificuldades, mas abrindo e construindo novas áreas de atuação, delimitando com maior clareza os espaços da saúde e da educação, sem perder a perspectiva de um trabalho interdisciplinar.

Tornou-se, assim, possível uma efetiva integração da equipe do PSE com as escolas, por meio de um trabal ho sistemático com professores da rede estadual de ensino fundamental. Esse trabalho, voltado para repensar a prática pedagógica nas primeiras séries, buscava estratégi as de atuação capazes de mobilizar o desejo de aprender, a auto-estima e a autonomia no processo de aprendizagem da criança. Em um relato do trabalho com pequenos grupos de crianças repetentes, essa preocupação se explicita na fala de um aluno e da educadora:

“Eu queria estudar... aí... eu vim aqui na esola. Primeiro... eu não gostava. Depois, eu fui acostumando. Daí, a genteficamos estudando... Folheando o livro a cada dia..." (Marcos, dez anos).

“A minha preocupação foi valorizar a voz das crianças - a voz queexpressa desejos, comunica idéias, constrói conhecimentos - dentro de uma prática lúdica, criativa e reflexiva" (Pereira, 1995:256).

O processo vivenciado pela equipe dentro de uma proposta interdisciplinar permitiu acei- tar o desafio de ampliar o programa, consolidando um espaço de referência para estágios de profissionais das áreas de saúde e educação. Assim, o PSE incorporou à sua atuação prática um trabalho de formação nas duas áreas em questão (Pereira,1993; Pereira et al., 1996), visando formar profissionais críticos, capazes de perceber a complexidade dos problemas da criança em processo de escolarização e o papel da equipe interdisciplinar no trabalho com o escolar.

A avaliação dessa experiência permitiu observar uma relação significativa entre o desenvolvimento dos estagiários nas escolas e a mobilização destas para mudanças na relação professor-aluno e escola-comunidade, no uso de propostas pedagógicas inovadoras, na valorização do próprio trabalho da escola e do professor.

Mais recentemente, novas demandas e proposições vêm-se formalizando por meio de outras parcerias com a comunidade e com estudantes da FMB em projetos de extensão universitária, voltados para uma atuação pedagógica nos campos da educação e da saúde.

Hoje o PSE constitui uma parceria entre a Secretaria Municipal de Saúde e Meio Ambiente de Botucatu e a Faculdade de Medicina de Botucatu, Departamento de Saúde Pública. Conta com a participação de profissionais de diversas instituições: Faculdade de Medicina de Botucatu, Departamento de Educação do IB/U NESP e Centro de Saúde Escola da UNESP, Secretaria de Saúde e Secretaria da Educação da Prefeitura Municipal de Botucatu.

\section{Construindo caminhos}

Com ênfase na dimensão pedagógica, têm-se buscado estratégias metodológicas que viabilizem a construção coletiva de conhecimentos nos campos da saú de e da educação. A possibilidade de abrir o projeto para estágios em diversas áreas de conhecimento, no interior das escola e nos centros de saúde, além de contribuir para uma formação crítica dos próprios envolvidos - estagiários e equipe do programa - tem propiciado algumas mudanças significativas nas escolas, mobilizando a comunidade escolar para repensar práticas e problematizar relações por meio de discussão e avaliação do trabalho do estagiário. Nos centros de saúde, que passam por um processo crítico, com grande ênfase no trabalho centrado na consulta médica, no 'modelo queixa-conduta' e no pronto-atendimento, o programa desperta a possibilidade de um outro modelo de trabalho, com maior envolvimento dos profissionais, com ações e atividades refl eti das e organizadas para 
intervenções mais efetivas. Para a comunidade de escolares e familiares, o programa tem significado uma chance de maior participação e de crédito no desenvolvimento dessas crianças.

A reflexão sobre essa experiência de atuação nas interfaces da saúde e da educação permitiu perceber a possibilidade de um trabalho

\section{Agradecimentos}

As autoras agradecem à equipe do Programa de Saúde Escolar de Botucatu e a seus estagiários a possibilidade de compartilhar a construção deste trabalho, bem como o apoio do Departamento de Saúde Pública da Faculdade de Medicina de Botucatu - UNESP e do Projeto UNI - Botucatu (Fundação Kellogg).

\section{Referências}

AYRES, J. R. C. M., 1990. Ad olescência e saúde coletiva: Aspectos epistemológi cos da abordagem programática. In: Programação em Saúde Hoje (L. B. Schraiber, org.), pp. 139-182, São Paulo: Editora Hucitec.

CASTELLANOS, P. L., 1991. Sistemas Nacionales de Vigilancia dela Situación de Salud según Condiciones deVida y del Impacto de las Acciones deSalud y Bienestar. Washington, DC: Organización Panamericana de la Salud. (mimeo.)

CECCIN, R. B.; BURD, E. G.; PETTRUCI, L. E. S.; SANTOS, N. \& SILVEIRA, R., 1992. Reflexão e ação pela criança e adolescente com vistas à saúde integral coletiva. In: Congresso Brasileiro de Saúde Coletiva, Resumos, Vol. 3, p. 33, Porto Alegre: Programa.

CYRINO, E. G., 1994. Estudo de um Programa de Saúde Escolar em uma Escola Estadual da Periferia de Botucatu, São Paulo. Dissertação de M estrado, São Paulo: Faculdade de Medicina, Universidades de São Paulo.

ELIAS, M. C. \& FELDM ANN, E., 1993. A busca da interdisciplinaridade e competência nas disciplinas dos cursos de Pedagogia. In: Práticas Interdisciplinares na Escola (I. C. A. Fazenda, org.), pp. 91-102, São Paulo: Cortez.

FERREIRO, E., 1986. Reflexões sobreAlfabetização. 5a Ed., São Paulo: Cortez.

FONSECA, L. E., 1988. O Aluno Carentee Repetente não éDoente. Dissertação de Mestrado, Rio de Janeiro: Escola Nacional de Saúde Pública, Fundação Oswaldo Cruz.

FREIRE, P. \& FAÚNDES, A., 1985. Por uma Pedagogia da pergunta. Rio de Janeiro: Paz e Terra.

GONÇALVES, R. B. M.; SCHRAIBER, L. B. \& NEMES, M. I. B., 1990. Seis teses sobre a ação programática em saúde. In: Programação em SaúdeHoje (L. B. Schraiber, org.), pp. 37-63, São Paulo: Editora Hucitec

LIMA, M. H., (s.d.). Atenção à Saúde da Criança na Rede Básica de Saúde - Críticas e Propostas. Campinas: ERSA. (mimeo.)

PATTO, M. H. S., 1990. A Produção do Fracasso Escolar. São Paulo: T. A. Queiroz. interdisciplinar, tendo como ponto de partida e de chegada a problematização da realidade, construindo coletivamente o saber, "ao buscar, juntos, o novo, o risco, a descoberta, o diálogo, a troca, o conhecer, deixando que cada um assumisse a sua própria prática dentro dos próprios limites" (Elias \& Feldmann, 1993:92).
PEREIRA, M. L. T., 1993. Eu não Gosto de Cópia. Reflexões sobre a Prática Alfabetizadora. Tese de Doutorado, São Paulo: Escola de Comunicações e Artes, Universidade de São Paulo.

PEREI RA, M. L. T., 1995. Histórias de Crianças: Uma Experiência com Artee Alfabetização. Artena Escola. São Paulo: Universidade Cruzeiro do Sul.

PEREIRA, M. L. T. \& CYRINO, E. G., 1996. A construção de parcerias na formação do professor. In: Congresso Estadual Paulista sobre Formação de Educadores, Anais, p.5, Águas de São Pedro: Universidade Estadual de São Paulo.

SMSSP (Secretaria Municipal de Saúde de São Paulo), 1990. Proposta para um Model o deAtenção à Saúde da Criança. São Paulo: SM SSP. (mimeo.)

SCHRAIBER, L. B., 1992. Epidemiologia em serviços: Uma tecnologia de que tipo? In: Congresso Brasileiro de Epidemiologia, Anais, Belo Horizonte: Abrasco. (mimeo.)

SCHRAIBER, L. B., 1990. Programação hoje: A força do debate. In: Programação em Saúde Hoje (L. B. Schraiber, org.), pp. 11-35, São Paulo: Editora Hucitec

SUCUPIRA, A. C. S. L.; MOYSÉS, M. A. \& NOVAES, H. M. D., 1986. O papel do pediatra nas dificuldades escolares. Pediatria, 8:23.

SUCUPIRA, A. C. S. L.; CAPUERI, P. F.; RANA, W.; ZUCOLOTTO, S. M. C.; GIORA, M. J.; ZARO, A. P. \& CARVALHO, P. R., 1989. A Saúde Escolar ea Atenção Integral à Saúde da Criança e do Adolescente. São Paulo: Secretaria Municipal de Saúde. (mimeo.)

URIBE-RIVERA, F. J., 1989. Planejamento eProgramação em Saúde Um EnfoqueEstratégico. São Paulo: Cortez.

VALLA, V. V. \& HOLLANDA, E., 1989. Fracasso escolar, saúde e cidadania. In: Demandas Populares, Políticas Públicas e Saúde(N. R. Costa, org.), pp. 103143, Petrópolis: Editora Vozes.

VYGOTSKY, L. S., 1987. A Formação Social da Mente. São Paulo: Martins Fontes.

YUNES, J.; CAMPOS, O. \& CARVALHO, V., 1987. Assistência à infância, à adolescência e à maternidade no Brasil. Boletín de la Oficina Sanitaria Panamericana, 103:33-42. 